\title{
Innovative data collection methods in criminological research: editorial introduction
}

\author{
Jean-Louis van Gelder ${ }^{{ }^{*}}$ and Stijn Van Daele ${ }^{2}$
}

\begin{abstract}
Novel technologies, such as GPS, the Internet and virtual environments are not only rapidly becoming an increasingly influential part of our daily lives, they also have tremendous potential for improving our understanding of where, when and why crime occurs. In addition to these technologies, several innovative research methods, such as neuropsychological measurements and time-space budgets, have emerged in recent years. While often highly accessible and relevant for crime research, these technologies and methods are currently underutilized by criminologists who still tend to rely on traditional data-collection methods, such as systematic observation and surveys. The contributions in this special issue of Crime Science explore the potential of several innovative research methods and novel technologies for crime research to acquaint criminologists with these methods so that they can apply them in their own research. Each contribution deals with a specific technology or method, gives an overview and reviews the relevant literature. In addition, each article provides useful suggestions about new ways in which the technology or method can be applied in future research. The technologies describe software and hardware that is widely available to the consumer (e.g. GPS technology) and that sometimes can even be used free of charge (e.g., Google Street View). We hope this special issue, which has its origins in a recent initiative of the Netherlands Institute for the Study of Crime and Law Enforcement (NSCR) called CRIME Lab, and a collaborative workshop together with the Research consortium Crime, Criminology and Criminal Policy of Ghent University, will inspire researchers to start using innovative methods and novel technologies in their own research.
\end{abstract}

\section{Issues and challenges facing crime researchers}

Applied methods of scientific research depend on a variety of factors that play out at least in part beyond researcher control. One such factor regards the nature and availability of data, which largely determines the research questions that can be addressed: Data that cannot be collected cannot serve to answer a research question. Although this may seem like stating the obvious, it has had and continues to have far-reaching implications for criminological research. Because crime tends to be a covert activity and offenders have every interest in keeping it that way, crime in action can rarely be observed, let alone in such a way as to allow for systematic empirical study. Consequently, our knowledge of the actual offending process is still limited and relies in large part on indirect evidence. The same applies to offender motivations and the offending process; these cannot be measured directly in ways similar to how

\footnotetext{
* Correspondence: jlvangelder@nscr.nl

${ }^{1}$ Netherlands Institute for the Study of Crime and Law Enforcement (NSCR), De Boelelaan 1105, 1081 HV Amsterdam, The Netherlands

Full list of author information is available at the end of the article
}

we can observe say the development of a chemical process or the workings of gravity. This has placed significant restrictions on the way crime research has been performed over the years, and most likely has coloured our view of crime and criminality by directing our gaze towards certain more visible elements such as offender backgrounds, demographics and criminal careers.

Another factor operating outside of researcher control regards the fact that the success of scientific analyses hinges on the technical possibilities to perform them. Even if researchers have access to particular data, this does not guarantee that the technology to perform the analysis in the best possible way is available to them. The complexity of the social sciences and the interconnectivity of various social phenomena, together with the lack of a controlled environment or laboratory, require substantial computational power to test highly complicated models. For instance, the use of space-time budgets, social network analysis or agent-based modelling can be quite resource-intensive. Adding the complexity of social reality to the resulting datasets (e.g. background variables related to social

\section{Springer}


structure, GIS to incorporate actual road networks, etc.) puts high pressure on the available computational power of regular contemporary desktop computers. Addressing these challenges has practical and financial consequences, which brings us to the third issue, which although more mundane in nature is by no means limited to the applied sciences: a researcher requires the financial and non-financial resources to be able to perform his research.

All three factors have a bearing on the contents and goals of the present special issue. More specifically, the methods that are discussed and explained in different articles in this special issue each address one or more of these factors and have, we believe, the potential to radically change the way we think about crime and go about doing crime research. The methods and technologies are available, feasible and affordable. They are, so to speak, there for the taking.

\section{Notes on criminological research methods}

The above factors have contributed to a general tendency within criminology to stick to particular research traditions, both in terms of method and analysis. Although these traditions are well-developed and have undergone substantial improvement over the decades, most changes in the way research is performed in the discipline have been of the 'more of the same' variety, and most involve gradual improvements, rather than embodying 'scientific revolutions' and 'paradigm shifts' that have radically changed how criminologists go about doing research and thinking about crime.

With respect to methodology, since the field's genesis in the early 20th century, research in criminology has largely spawned studies using similar methods. In a metaanalysis of articles that have appeared in seven leading criminology and criminal justice journals in 2001-2002, Kleck et al. (2006) demonstrate that survey research is still the dominant method of collecting information (45.1\%), followed by the use of archival data (31.8\%), and official statistics (25.6\%). Other methods, such as interviews, ethnographies and systematic observation, each account for less than $10 \%$ (as methods are sometimes combined the percentages add up to more than 100\%). Furthermore, the Kleck et al. meta-analysis shows that as far as data analysis is concerned, nearly three quarters $(72.5 \%)$ of the articles published uses some type of multivariate statistics. As such, it appears that criminology has developed a research tradition that started to dominate the field, though not to everybody's enthusiasm. Berk (2010), for example, states that regression analysis, in its broad conception, has become so dominant that researchers give it more credit than they sometimes should and take some of its possibilities for granted. Whereas certain research traditions may have a strong added value, they run the risk of being used more out of habit than for being the most appropriate method to answer a particular research question. As innovation influences our daily lives and how we perform our daily activities, there is no reason why it should not influence our way of performing science as well.

\section{Innovation and technology in daily life and their application in criminological research}

Novel technologies, such as GPS, the Internet, and virtual environments are rapidly establishing themselves as ingrained elements of our daily lives. Think, for example, of smart phones. While unknown to most of us as recently as ten years ago, today few people would leave their home without carrying one with them. Through their ability to, for example, transmit visual and written communication, use social media, locate ourselves and others through apps and GPS, smart phone technology has radically changed the way we go about our daily routines. The research potential of smart phone technology for improving our understanding of social phenomena is enormous and includes a better understanding of where, when and why crime occurs through automated data collection, interactive tests and surveys (Miller 2012). Yet, so far surprisingly few crime researchers have picked up on the possibilities this technology has to offer.

A similar point can be made with respect to the Internet. While most researchers have made the transition from paper-and-pencil questionnaires to online surveys and online research panels may have become the norm for collecting community sample data, much of the potential of the Internet for crime research remains untapped. Think, for example, of the possibility of using Google Maps and Google Street View for studying spatial aspects of crime and the ease with which we can virtually travel down most of the roads and streets to examine street corners, buildings and neighbourhoods. The launch of Google Street View in Belgium stirred up controversy because it was perceived by police officers as a catalogue for burglars and was expected to lead to an increase in the number of burglaries. While it is not clear whether this has actually happened, crime studies reporting these tools in their method sections are few and far between.

In addition to novel technologies, several innovative research methods, such as social network analysis, timespace budgets, and neuropsychological measures that can tap into people's physiological and mental state, have emerged in recent years and have become common elements of the tool-set used in some of criminology's sister disciplines, such as sociology, psychology and neuroscience. Again, while often highly accessible and relevant for crime research, these methods are currently underutilized by crime researchers. The promising and emerging field of 
neurocriminology is an important exception in this respect (see e.g., Glenn \& Raine 2014).

To revert back to the three issues that have restricted the gaze of criminology mentioned at the outset of this article: accessibility of data, type of data analysis, and availability of resources, the new methods and technologies seem to check all the boxes. Much more data have become accessible for analysis through novel technologies, new methods for data-analysis and data-collection have been developed, and most of it is available at the consumer level. In other words, this allows for collecting and examining data in ways that go far beyond what we are used to in crime research. For example, methods combining neurobiological measurements with virtual environments can tell us a lot about an individual's mental and physical states and how they go about making criminal decisions, which were until recently largely black boxes. Finally, novel technologies are often available on the consumer market, such as smart phones, tablets, and digital cameras, and require only very modest research budgets. In some cases, such as the automated analysis of footage made by security cameras, simulations, or the use of honeypots that lure cyber criminals into hacking them, they even allow for systematically examining crime in action.

\section{Goals of this special issue of Crime Science}

The principal aim of the present special issue is to acquaint crime researchers and criminology students with some of these technologies and methods and explain how they can be used in crime research. While widely diverging, what these technologies and methods have in common is their accessibility and potential for furthering our understanding of crime and criminal behavior. The idea, in other words, is to explore the potential of innovative research methods of data-collection and novel technologies for crime research and to acquaint readers with these methods so that they can apply them in their own research.

Each article deals with a specific technology or method and is set-up in such a way as to give readers an overview of the specific technology/method at hand, i.e., what it is about, why to use it and how to do so, and a discussion of relevant literature on the topic. Furthermore, when relevant, articles contain technical information regarding the tools that are currently available and how they can be obtained. Case studies also feature in each article, giving readers a clearer picture of what is at stake. Finally, we have encouraged all contributors to give their views on possible future developments with the specific technology or method they describe and to make a prediction regarding the direction their field could or should develop. We sincerely hope this will inspire readers to apply these methods in their own work.

While not intended as a manual, after reading an article, readers should not only have a clear view of what the method/technology entails but also of how to use it, possibly in combination with one or more of the references that are provided in the annotated bibliography provided at the end of each article. The contributions may also be useful for researchers already working with a specific method or students interested in research methodology.

\section{Article overview}

Hoeben, Bernasco and Pauwels discuss the space-time budget method. This method aims at retrospectively recording on an hour-by-hour basis the whereabouts and activities of respondents, including crime and victimization. The method offers a number of advantages over existing methods for data-collection on crime and victimization such as the possibility of studying situational causes of crime or victimization, possibly in combination with individual lifestyles, routine activities and similar theoretical concepts. Furthermore, the space-time budget method offers the possibility of collecting information on spatial location, which enables the study of a respondent's whereabouts, going beyond the traditional focus of ecological criminological studies on residential neighborhoods. Finally, the collected spatial information on the location of individuals can be combined with data on neighborhood characteristics from other sources, which enables the empirical testing of a variety of ecological criminological theories.

The contribution of Gerritsen focuses on the possibilities offered by agent-based modeling, a technique borrowed from the domain of Artificial Intelligence. Agent-based modeling $(\mathrm{ABM})$ is a computational method that enables a researcher to create, analyse and experiment with models composed of agents that interact within a computational environment. Gerritsen explains how modeling and simulation techniques may help gain more insight into (informal) criminological theories without having to experiment with these phenomena in the real world. For example, to study the effect of bystander presence on norm violation, an 'artificial neighborhood' can be developed that is inhabited by 'virtual aggressors and victims', and by manipulating the agents' parameters different hypothetical scenarios may be explored. This enables criminologists to investigate complex processes in a relatively fast and cheap manner.

Since the pioneering early studies of the 1990s hinted at its promise as a method for social scientific research, Virtual Reality (VR) technology is increasingly used as a research tool by social scientists from various disciplines such as psychology and sociology. Given recent developments that have greatly enhanced the realism, reduced costs, and increased possibilities for application, it seems well on its way to become an established method in the social sciences. In their contribution Van Gelder, Luciano and Otte explore the potential of VR as a tool for research in criminology. Their article offers a description 
of how virtual reality has been used in social scientific research before discussing the potential it holds for criminology. By way of illustration, the authors describe a recent case study that has used immersive virtual reality for theory testing, which gives an idea of the unique possibilities of VR for studying crime.

Cornet also draws from criminology's sister disciplines in her article on neurobiological measurements observing that while the literature on the relationship between neurobiological factors and antisocial behavior has accumulated in recent years, the use of neurobiological measurements does not yet play a central role in criminological research. One of the underlying reasons Cornet identifies is the idea among criminologists that it is complicated to use these techniques compared to sociological, psychological and behavioral instruments. This article aims to provide an overview of the most commonly used neurobiological measurements, their added value and, importantly, how they can be relatively easily implemented in criminological research.

Vandeviver explains the possibilities and merits of online mapping applications such as Google Maps and Google Street View. While these technologies have evoked concern from law enforcement agencies that offenders could exploit them, Vandeviver notes that for criminologists they also open up several new perspectives to conduct research. Elaborating on results from prior studies in related fields and personal research experience, three potential uses of Google Maps and Google Street View in criminological research are explored and discussed. In particular, this article deals with how these online mapping applications can generate new research questions, how they allow revisiting and re-assessing established theories in criminology and common research practices, and how they should be considered an essential part of the methodological toolkit of criminologists.

Lemieux, also taking a spatial approach, describes how digital cameras with integrated GPS units can be used to collect useful data for criminological purposes observing that while visual observations have traditionally been an important method of criminological research, when combined with spatial data, such observations become a particularly useful way to describe how crime and disorder are distributed. Lemieux describes the utility and ease of using geo-tagged photographs and GPS tracks for monitoring illegal activity in urban and rural settings presenting case studies from Uganda and Amsterdam.

Vander Laenen discusses a method of qualitative criminological research, the Nominal Group Technique (NGT), which is not necessarily innovative by and of itself, but which has hardly been applied in criminology. The NGT is a highly structured technique with characteristics of an individual survey and a focus group. However its structure limits the influence of the researcher and of group dynamics and guarantees equal participation to all group members and equal influence of (conflicting) values and ideas. The NGT forms an ideal technique for gathering and prioritizing ideas and for innovative solution planning. Furthermore, despite the structure of the procedure, the technique can be modified and easily be applied in a mixed-methods research design. By means of an example, Vande Laenen reports on the use of the NGT in a study on the needs assessment of drug (prevention) policy.

In the final contribution to this special issue on innovative methods of data collection in criminology, De Bondt suggests solutions for doing cross-border criminal policy research and the confusion in wording and interpretation that emerge when comparing different legal systems. Correctly interpreting foreign information and using it as a valid source to substantiate analyses on where, when and why crime occurs and/or how to improve the fight against crime, often requires the expertise of people that are very familiar with a certain legal system. Based on a literature review and a number of successful experiences with the method in past research projects, the strengths and weaknesses of working with (predominantly) multiple choice based questionnaires sent to a single point of contact in each of the EU member states is elaborated upon.

\section{Competing interests}

The authors declare that they have no competing interests.

\section{Authors' contributions}

Both Jean-Louis van Gelder and Stijn van Daele contributed to all aspects of the paper. Both authors read and approved the final manuscript.

\section{Author details}

${ }^{1}$ Netherlands Institute for the Study of Crime and Law Enforcement (NSCR), De Boelelaan 1105, 1081 HV Amsterdam, The Netherlands. ${ }^{2}$ Ghent University, Universiteitstraat 4, 9000, Ghent, Belgium.

Received: 17 April 2014 Accepted: 30 April 2014

Published online: 12 August 2014

\section{References}

Berk, R. (2010). What you can and can't properly do with regression. J Quant Criminol, 26(4), 481-487. doi:10.1007/s10940-010-9116-4.

Glenn, AL, \& Raine, A. (2014). Neurocriminology: implications for the punishment, prediction and prevention of criminal behaviour. Nature Reviews Neuroscience, 15(1), 54-63. doi:10.1038/Nrn3640.

Kleck, G, Tark, J, \& Bellows, J. (2006). What methods are most frequently used in research in criminology and criminal justice? [Article]. J Crim Justice, 34(2), 147-152. doi:10.1016/j.jcrimjus.2006.01.007.

Miller, G. (2012). The smartphone psychology manifesto. Perspect Psychol Sci, 7(3), 221-237. doi:10.1177/1745691612441215.

doi:10.1186/s40163-014-0006-1

Cite this article as: van Gelder and Van Daele: Innovative data collection methods in criminological research: editorial introduction. Crime Science 2014 3:6 